Norman, Sylvia

[64]

\section{Analysis of TP53 mutations in human malignant gliomas}

\author{
Sylvia Norman ${ }^{1}$, Adrienne Scheck ${ }^{2}$ \& Jeff Braman ${ }^{1}$ \\ ${ }^{1}$ Stratagene, La Jolla, California, USA \\ ${ }^{2}$ Barrow Neurological Institute, Phoenix, Arizona, USA
}

TP53 is involved in the regulation of the cell cycle, response to DNA damage and induction of apoptosis. Mutations in this gene are thought to contribute to the development, progression or both of a variety of tumors, including human malignant glioma. Molecular analyses of primary malignant gliomas have demonstrated cellular heterogeneity in gene loss, rearrangement, amplification, transcription and translation. Furthermore, the rapid recurrence of this tumor demonstrates the presence of cells that are intrinsically resistant to therapy. Whether mutations in TP53 contribute to therapy resistance and tumor recurrence in malignant gliomas is not known. To determine if TP53 mutations are indicative of cells with a selective advantage that contributes to the survival and/or recurrence of glial tumors, we undertook a study to compare matched cells from primary and recurrent tumors from the same patients. We will present results from our analysis of TP53 mutations present in cells derived from both primary and recurrent glial tumors using polymerase chain reaction and sequence analysis to screen for mutations in exons 5-8 of TP53.

Novoradovskaya, Natalia

\section{Using universal human reference RNA in microarray gene expression studies}

Natalia Novoradovskaya ${ }^{1}$, Nicki Chin ${ }^{2}$, Terry Payette ${ }^{1}$ Alexander Pergamenschikov², Michael Fero ${ }^{2}$, David Botstein ${ }^{2}$ \& Jeff Braman ${ }^{1}$

\section{${ }^{1}$ Stratagene, La Jolla, California, USA}

${ }^{2}$ Stanford University, Stanford, California, USA

The expression profiles of thousands of genes can be analyzed simultaneously by hybridizing labeled complementary DNA targets to microarrays. Two-color hybridization allows comparison of two samples labeled with different fluorescent dyes on the same microarray. Normalized signals are expressed as the ratio of signals from Cy5 experimental and $\mathrm{Cy} 3$ reference probes. Use of a common reference sample across multiple experiments provides reliable data comparison. A reference sample should provide a hybridization signal at as many probe elements as possible. We chose 21 human cell lines, representing different tissues, to approximate the expression profile of all human genes. The expression profile of each individual cell line and that of a pool of RNAs from 21 cell lines were compared. Hybridization was performed using $24 \mathrm{~K}$ probe element human microarrays (Stanford University) and GeneConnection Discovery microarrays (Stratagene). Signal distribution on these microarrays demonstrates that reference cDNA hybridizes to a larger number of tethered probes than cDNA from any single cell line. Ten different cell lines were chosen for Universal Human Reference RNA, providing optimal coverage on human microarrays; these can be used as a reference sample for any microarray experiment.
Nuchtern, Jed

[66]

\section{cDNA microarray analysis of differential gene expression in MYCN-amplified neuroblastoma tumor cells}

Jed Nuchtern ${ }^{1}$, Jason Shohet ${ }^{1}$, Susan Stuart ${ }^{2}$, M. John Hicks ${ }^{1}$ \& Susan Burlingame ${ }^{1}$

${ }^{1}$ Baylor College of Medicine, Houston, Texas, USA

${ }^{2}$ Incyte Genomics, Palo Alto, California, USA

Neuroblastoma is a common pediatric solid tumor that is frequently incurable. Biological risk stratification implicates amplification of the $M Y C N$ oncogene as a robust marker for poor prognosis. We have undertaken an analysis of gene expression in $M Y C N$-amplified and non-amplified neuroblastoma tumors based on complementary DNA microarrays to advance our knowledge of the pathogenesis of this aggressive disease. We isolated messenger RNA from tumor cell primary cultures of two INSS stage 4 neuroblastoma patients, one with MYCN amplification and the other with normal MYCN copy number. Relative gene expression was determined after hybridization of the fluorophore-labeled cDNAs to a human cDNA microarray representing over 6,800 unique expressed genes. Using a differential gene expression ratio of 3.5 as a screening criterion, we identified 370 unique genes for further analysis. Of these, 78 were upregulated in the $M Y C N$ amplified neuroblastoma and 292 had decreased expression. Amplification correlated with increased transcript levels of genes associated with cell proliferation, including cell cycle regulatory genes such as $C D C 2$ and $C D C 28$ protein kinase, and the Ki-67 antigen, as well as many other annotated molecules not previously linked to $M Y C N$ amplification and several uncharacterized expressed sequence tag sequences. A coherent group of genes was downregulated in the $M Y C N$-amplified cells, including the genes for the class I and class II major histocompatibility complex antigens, the gene for peptide antigen transporter TAP1, and several interferon responsive transcripts. MYCN amplification correlates with a distinct gene expression pattern in neuroblastoma that is consistent with the clinical phenotype of these tumors. Further characterization of this motif should enable the development of new therapies for these difficult tumors.

O’Connor, Maureen

[67]

\section{Identification of genes that are differentially expressed in response to EGF receptor signaling in A549 lung cancer cells using a combination of suppression subtractive hybridization and microarrays}

\author{
Maureen O'Connor, Alain Guimond, Christiane Cantin \& \\ Joanne Magoon
}

Biotechnology Research Institute, Montreal, Quebec H4P 2R2, Canada

Since current treatments for almost all patients with non-small-cell lung cancer are not satisfactory, and since prognosis is adversely affected by the overexpression of EGF receptors, we are attempting to identify new genes whose expression is modulated by the EGF signaling pathway in A549 lung carcinoma cells. The cells were treated with either EGF or C225 (a neutralizing antibody that blocks the EGF receptor). We have shown that these treatments affect the epithelial-to-mesenchymal transdifferentiation of these cells. We prepared complementary DNA libraries from EGF-treated, C225Ab-treated or control cells and subjected them to suppression subtractive hybridization to identify both up- and downregulated genes. These enriched cDNAs were fluorescently labeled and used to screen 9,600 human 